European Conference on Laboratory Astrophysics - ECLA

C. Stehlé, C. Joblin and L. d'Hendecourt (eds)

EAS Publications Series, 58 (2012) 295-299

www.eas.org

\title{
COMBINING TWO VUV LASERS WITH THE TIME-SLICED VELOCITY MAPPED ION IMAGING METHOD FOR STUDYING THE PHOTODISSOCIATION OF SMALL MOLECULES
}

\author{
Y. Song ${ }^{1}$, H. Gao ${ }^{1}$, C.Y. $\mathrm{Ng}^{1}$ and W.M. Jackson ${ }^{1}$
}

\begin{abstract}
A new experimental setup is described that allows us to study the state-selected photodissociation dynamics of small molecules of astrophysical interest using two independently tunable VUV radiation sources.
\end{abstract}

\section{Introduction}

We have previously reported on the vacuum ultraviolet (VUV) photodissociation of small molecules of astrophysical interest, i.e., $\mathrm{N}_{2}, \mathrm{NO}, \mathrm{CO}$ and $\mathrm{CO}_{2}$, using time-sliced velocity-mapped ion imaging (TSVMI) combined with one-color VUV radiation (Pan et al. 2011; Gao et al. 2011; Gao et al. 2011; Gao et al. 2012). These studies were performed in the $\mathrm{H}$ I energy below $13.6 \mathrm{eV}$ and the branching ratios for the various product channels were determined at different dissociation wavelengths. It was found that long-lived metastable states of $\mathrm{N}\left({ }^{2} \mathrm{D}\right), \mathrm{O}\left({ }^{1} \mathrm{D}\right)$, and $\mathrm{C}\left({ }^{1} \mathrm{D}\right)$ are produced with large amounts of translational energy. These long-lived energetic atoms can profoundly affect the chemistry of interstellar clouds, circumstellar envelopes, comets and planetary atmospheres. Recently our experimental setup was greatly improved by adding an additional tunable VUV system in order to study the state-selected photodissociation dynamics of molecules. The current experimental apparatus is described here, with emphasis on its advantages.

\section{Experimental}

A detailed description of TSVMI apparatus can be found in Zhou et al. (2006). The two tunable VUV sources are produced by resonant four-wave mixing (RFWM) that produces VUV light at $2 \omega_{1}+\omega_{2}$ or $2 \omega_{1}-\omega_{2}$ mixing scheme using Kr gas

1 Department of Chemistry, University of California, Davis, CA 95616, USA;

e-mail: wmjackson@ucdavis.edu 


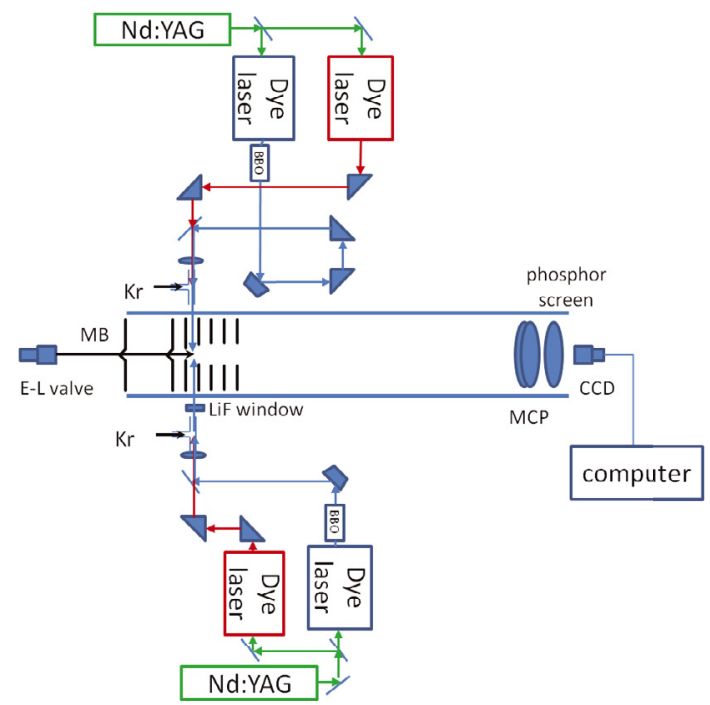

Fig. 1. Time-sliced velocity-mapped ion-imaging apparatus combined with two RFWMVUV laser sources.

as the nonlinear medium (Mahon \& Tomkins 1982; Hilbig \& Wallenstein 1983; Hilbig \& Wallenstein 1982). The typical energies for $\omega_{1}$ and $\omega_{2}$ are $1.0 \mathrm{~mJ} / \mathrm{pulse}$ and $5 \mathrm{~mJ} /$ pulse, respectively. The light includes frequencies corresponding to $2 \omega_{1}+\omega_{2}, 2 \omega_{1}-\omega_{2}, 3 \omega_{1}, \omega_{1}$ and $\omega_{2}$. The $2 \omega_{1}+\omega_{2}$ and $3 \omega_{1}$ can be blocked by inserting a moveable LiF window with cutoff wavelength at $105 \mathrm{~nm}$. The combination of the TSVMI apparatus with the two VUV lasers is illustrated in Figure 1. An Even-Lavie valve (EL-5-2004, nozzle diameter $0.02 \mathrm{~mm}$ ) operating at $30 \mathrm{~Hz}$ with a stagnation pressure of 30-50 psi produces a pulsed supersonic molecular beam. The beam is collimated by two conical skimmers prior to entering the interaction region along the axis of velocity-mapped ion imaging optics where it is crossed by the two perpendicular VUV sources that are counter-propagating to each other, one to photodissociate, $\mathrm{PD}$, the parent molecule, and the other to photoionize, PI, the product atoms. The time delay between the PD and PI sources is generally set to $20 \mathrm{~ns}$ to optimize the ion signal. The ion imaging optics set consists of six circular aperture lenses with adjustable voltages which allow the ions originating from a large volume with the same velocity to be focused on the same position of the imaging detector. The distance between the Even-Lavie nozzle and the interaction region is $15 \mathrm{~cm}$. The ions formed in the interaction region are accelerated by the ion imaging optics and after flying down the $75 \mathrm{~cm}$ flight tube they then strike a dual microchannel plates (MCP) detector with an active diameter of $75 \mathrm{~mm}$. Electrons from this MCP detector hit a P47 phosphor screen. The back MCP is normally set at $+1,100 \mathrm{~V}$ while a high voltage pulse is applied to the front MCP at the arrival time of ion of interest to achieve the ion slicing. The image formed on the phosphor screen is recorded by the DaVis CCD 


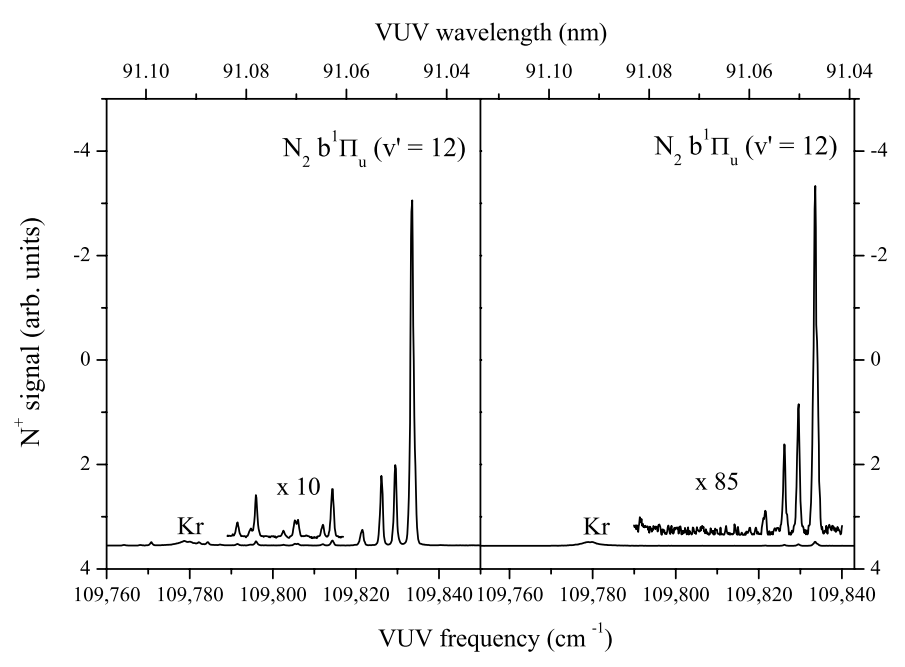

Fig. 2. PHOFEX spectra of $\mathrm{N}\left({ }^{2} \mathrm{D}\right)$ from the photodissociation of $\mathrm{N}_{2}$ molecule as a function of dissociation frequency from $109,760 \mathrm{~cm}^{-1}$ to $109,840 \mathrm{~cm}^{-1}$. Two VUV sources are used, one for PD and one for PI. In the left panel the PI VUV source is tuned to an autoionization line of $\mathrm{N}\left({ }^{2} \mathrm{D}\right)$ at $110,305 \mathrm{~cm}^{-1}$, while in the right panel the PI VUV source is tuned to $110,310 \mathrm{~cm}^{-1}\left(5 \mathrm{~cm}^{-1}\right.$ away from $\mathrm{N}\left({ }^{2} \mathrm{D}\right)$ autoionization line).

camera and the output of the camera is sent to a computer to be accumulated. When the photofragment excitation (PHOFEX) spectrum is being measured a dc voltage of $-1,400 \mathrm{~V}$ is applied to the front MCP and the back MCP is grounded. The ion signals from the back of the MCP detector are amplified fed into a digital oscilloscope and then into boxcar integrator. The PHOFEX spectrum is obtained by gating the appropriate ion signal while scanning the PD wavelength.

\section{Results}

Figure 2 shows the PHOFEX spectrum of $\mathrm{N}_{2}$ obtained by monitoring the $\mathrm{N}\left({ }^{2} \mathrm{D}\right)$ product from the photodissociation of $\mathrm{N}_{2}$, with the probe laser tuned on- and off-autoionization line of the $\mathrm{N}\left({ }^{2} \mathrm{D}\right)$ atom. This figure shows that when the probe laser is tuned to the autoionization line of the atom the PHOFEX spectrum is 85 times stronger. Lines that are buried in the noise in the right panel of Figure 2 are clearly observable with a good signal to noise ratio in the left panel. Branching ratios can also be determined with no correction for the ionization cross section.

(a) Direct ionization

Figure 3 demonstrates the sliced raw image, total kinetic energy release (KER) and angular distribution spectra of photodissociation of $\mathrm{N}_{2}$ molecule at $109,833.6 \mathrm{~cm}^{-1}$, accidentally overlapped $\mathrm{R}(0), \mathrm{R}(1)$ and $\mathrm{R}(2)$ lines via $\mathrm{b}^{1} \Pi_{u}$ $\left(\mathrm{v}^{\prime}=12\right) \leftarrow \mathrm{X}^{1} \Sigma_{g}+\left(\mathrm{v}^{\prime \prime}=0\right)$, which correspond to the strongest peak in Figure 2 (right panel). Both of PD and PI lasers (tuned to $110,310 \mathrm{~cm}^{-1}$ ) could directly 

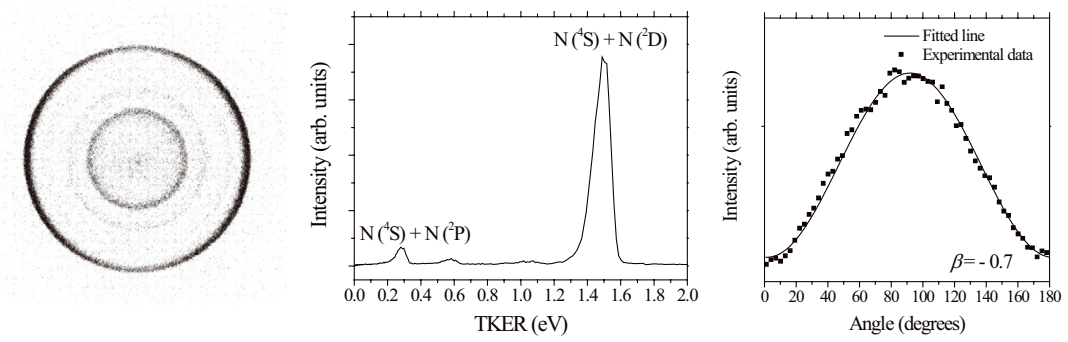

Fig. 3. Sliced raw image, total kinetic energy release and angular distribution spectra of photodissociation of $\mathrm{N}_{2}$ molecule at $\mathrm{R}(0), \mathrm{R}(1)$ and $\mathrm{R}(2)$ lines via $\mathrm{b}^{1} \Pi_{u}\left(\mathrm{v}^{\prime}=12\right)$ $\leftarrow \mathrm{X}^{1} \Sigma_{g}^{+}\left(\mathrm{v}^{\prime \prime}=0\right)$. PD laser was tuned to $109,833.6 \mathrm{~cm}^{-1}$ and PI laser was tuned to $110,310 \mathrm{~cm}^{-1}$, both of which directly ionize the products $\mathrm{N}\left({ }^{2} \mathrm{D}\right)$ and $\mathrm{N}\left({ }^{2} \mathrm{P}\right)$.
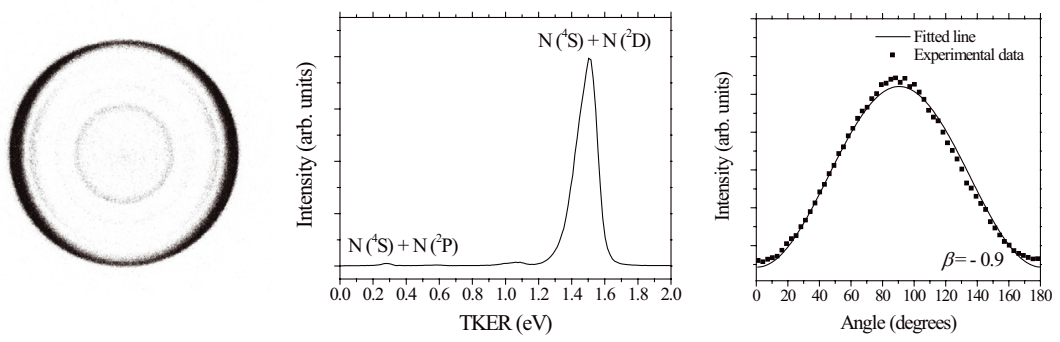

Fig. 4. Same as Figure 3 but with PI laser tuned to the autoionization level of $N\left({ }^{2} \mathrm{D}\right)$ at $110,305 \mathrm{~cm}^{-1}$ with an ionization cross section much larger than the direct ionization.

ionize $\mathrm{N}$ atoms. The predominate product channel is $\mathrm{N}\left({ }^{4} \mathrm{~S}\right)+\mathrm{N}\left({ }^{2} \mathrm{D}\right)$ with minor $\mathrm{N}\left({ }^{4} \mathrm{~S}\right)+\mathrm{N}\left({ }^{2} \mathrm{P}\right)$ participated.

\section{(b) Autoionization}

Figure 4 shows the sliced raw image, total KER and angular distribution spectra from photodissociation of $\mathrm{N}_{2}$ at the same ro-vibronic levels as in Figure 3. The experimental conditions were maintained the same as that of Figure 3 but PI laser was tuned to the autoionization level of $\mathrm{N}\left({ }^{2} \mathrm{D}\right)$ at $110,305 \mathrm{~cm}^{-1}$. Comparisons between the signal sizes in Figure 2 show that the autoionization cross section is approximately 85 times larger than the direct ionization cross section. The signal to noise ratio is much better than the previous case which could help to reduce the image accumulation time.

\section{(c) REMPI}

The VUV probe beam could be tuned to a resonance line of the $\mathrm{N}\left({ }^{4} \mathrm{~S}\right)$ atom and ionize it when it absorbs a second UV photon. Figure 5 shows the photodissociation of $\mathrm{N}_{2}$ at the same ro-vibronic levels as in Figures 3 and 4 . The PI laser was tuned to $110,323 \mathrm{~cm}^{-1}$ to coincide with $\mathrm{N}\left({ }^{4} \mathrm{~S}\right)$ transition $2 s^{2} 2 p^{2}\left({ }^{3} \mathrm{P}\right) 4 d^{4} \mathrm{P}_{3 / 2}$ 

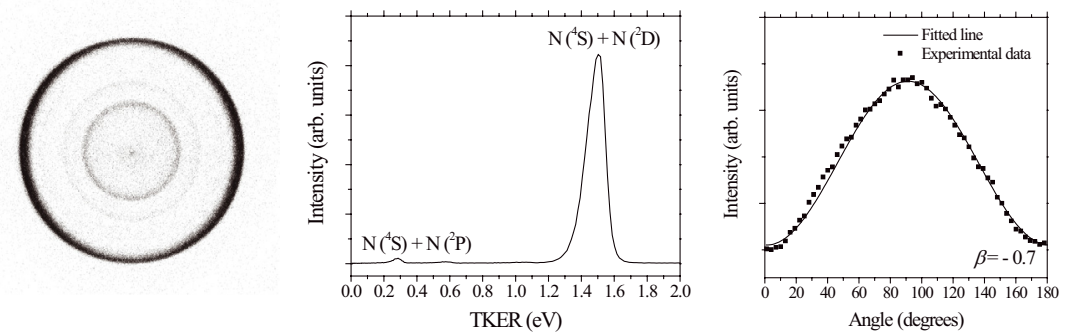

Fig. 5. Sliced raw image, total kinetic energy release and angular distribution spectra of photodissociation of $\mathrm{N}_{2}$ molecule at $\mathrm{R}(0), \mathrm{R}(1)$ and $\mathrm{R}(2)$ lines via $\mathrm{b}^{1} \Pi_{u}\left(\mathrm{v}^{\prime}=12\right) \leftarrow$ $\mathrm{X}^{1} \Sigma_{g}^{+}\left(\mathrm{v}^{\prime \prime}=0\right)$. PI laser was tuned to $110,323 \mathrm{~cm}^{-1}$ to coincide with $\mathrm{N}\left({ }^{4} \mathrm{~S}\right)$ transition $2 s^{2} 2 p^{2}\left({ }^{3} \mathrm{P}\right) 4 d{ }^{4} \mathrm{P}_{3 / 2} \leftarrow 2 s^{2} 2 p^{3}{ }^{4} \mathrm{~S}_{3 / 2}^{\circ}$.

$\leftarrow 2 s^{2} 2 p^{3}{ }^{4} \mathrm{~S}^{\circ}{ }_{3 / 2}$, which allows to detect two product channels, $\mathrm{N}\left({ }^{4} \mathrm{~S}\right)+\mathrm{N}\left({ }^{2} \mathrm{D}\right)$ and $\mathrm{N}\left({ }^{4} \mathrm{~S}\right)+\mathrm{N}\left({ }^{2} \mathrm{P}\right)$, at the same time but the signal to noise ratio of the $\mathrm{N}\left({ }^{2} \mathrm{D}\right)$ angular distribution is not quite as good as it using the autoionization line.

\section{Conclusion}

We have shown that the addition of a second VUV probe laser to our experimental setup increases the flexibility of the apparatus and enhances the signal to noise ratio for the detection of the products. This will allow us to extend our photodissociation studies at a variety of wavelengths for molecules such as $\mathrm{CO}, \mathrm{CO}_{2}$ and NO. Since the sensitivity for detection is also increased, there is a very good possibility that unstable free radicals such as $\mathrm{C}_{2}, \mathrm{C}_{3}$, etc. can also be investigated.

\section{References}

Gao, H., Yang, L., Pan, Y., et al., 2011, J. Chem. Phys., 135, 134319

Gao, H., Song, Y., Yang, L., et al., 2011, J. Chem. Phys., 135, 221101

Gao, H., Pan, Y., Yang, L., et al., 2012, J. Chem. Phys., 136, 134302

Hilbig, R., \& Wallenstein, R., 1982, IEEE J. Quantum Electon., 19, 1759

Hilbig, R., \& Wallenstein, R., 1983, IEEE J. Quantum Electon., 19, 194

Mahon, R., \& Tomkins, F., 1982, IEEE J. Quantum Electon., 18, 913

Pan, Y., Gao, H., Yang, L., et al., 2011, J. Chem. Phys., 135, 071101

Zhou, J., Lau, K-C., Hassanein, E., et al., 2006, J. Chem. Phys., 124, 034309 